\title{
STRICTLY POSITIVE SOLUTIONS FOR ONE-DIMENSIONAL NONLINEAR PROBLEMS INVOLVING THE $p$-LAPLACIAN
}

\author{
U. KAUFMANN ${ }^{\bowtie}$ and I. MEDRI
}

(Received 27 February 2013; accepted 2 July 2013; first published online 6 September 2013)

\begin{abstract}
Let $\Omega$ be a bounded open interval, and let $p>1$ and $q \in(0, p-1)$. Let $m \in L^{p^{\prime}}(\Omega)$ and $0 \leq c \in L^{\infty}(\Omega)$. We study the existence of strictly positive solutions for elliptic problems of the form $-\left(\left|u^{\prime}\right|^{p-2} u^{\prime}\right)^{\prime}+$ $c(x) u^{p-1}=m(x) u^{q}$ in $\Omega, u=0$ on $\partial \Omega$. We mention that our results are new even in the case $c \equiv 0$.

2010 Mathematics subject classification: primary 34B15; secondary 34B18, 35J25, $35 \mathrm{~J} 61$.

Keywords and phrases: elliptic one-dimensional problems, indefinite nonlinearities, $p$-Laplacian, strictly positive solutions.
\end{abstract}

\section{Introduction}

For $a<b$, let $\Omega:=(a, b)$, and let $p>1$ and $q \in(0, p-1)$. Let $m \in L^{p^{\prime}}(\Omega)$ and $0 \leq c \in$ $L^{\infty}(\Omega)$. Our aim in this paper is to study the existence of solutions for problems of the form

$$
\begin{cases}-\left(\left|u^{\prime}\right|^{p-2} u^{\prime}\right)^{\prime}+c(x) u^{p-1}=m(x) u^{q} & \text { in } \Omega \\ u>0 & \text { in } \Omega \\ u=0 & \text { on } \partial \Omega .\end{cases}
$$

For applications we refer to [4] and the references therein.

When $c \equiv 0$ and $0 \not \equiv m \geq 0$ it is known that (1.1) admits a solution. See, for example, [5, Theorem 5.1], or [2] and its references for the case $p=2$. On the other hand, allowing $m$ to change sign and under the assumption that $m(x) \geq m_{0}>0$ in some $\overline{\Omega^{\prime}} \subset \Omega$, it can be proved that the problem

$$
\begin{cases}-\left(\left|u^{\prime}\right|^{p-2} u^{\prime}\right)^{\prime}=m(x) u^{q} & \text { in } \Omega, \\ u=0 & \text { on } \partial \Omega\end{cases}
$$

possesses a nontrivial nonnegative solution (see [5, Theorem 5.2], or [1, Section 5]). We note however that in general a (nontrivial) nonnegative solution of (1.2) need not

This research was partially supported by Secyt-UNC.

(C) 2013 Australian Mathematical Publishing Association Inc. 0004-9727/2013 \$16.00 
be strictly positive in $\Omega$ (in contrast to the superlinear case), and that in fact the matter of existence of strictly positive solutions for these types of problems is quite intriguing.

Recently, several noncomparable sufficient conditions for the existence of strictly positive solutions for (1.2) were exhibited in [9] under some evenness assumptions on $m$ in the case $p=2$, and an extension of some of these results for a (linear) strongly uniformly second-order elliptic operator was given in the paper 'Strictly positive solutions for one-dimensional nonlinear elliptic problems', which has been submitted for publication by the current authors. We refer to it later as [KM].

Let us mention that a natural way to attack these kinds of problems is the wellknown sub and supersolution method. Moreover, it is quite simple to provide arbitrarily large supersolutions (see Remark 2.1 below). To construct the strictly positive subsolutions we shall adapt and extend the approach developed in [9] and [KM]. Roughly speaking, we shall divide $\Omega$ in parts, construct 'subsolutions' for each of them and then find conditions on $m, c, p$ and $q$ that guarantee that they can be joined accordingly to obtain the desired subsolution. Certain conditions are presented in Theorem 3.1, and assuming that $m^{-}$is essentially bounded, further noncomparable conditions are proved in Theorem 3.3 and Corollary 3.5.

Let us finally point out that although for the sake of simplicity we assume that $c \geq 0$, similar results can be obtained under some additional assumptions if $c$ changes sign in $\Omega$ (see Remark 3.6).

\section{Preliminaries}

It is well known that for $g \in L^{1}(\Omega)$, the problem $-\left(\left|u^{\prime}\right|^{p-2} u^{\prime}\right)^{\prime}=g$ in $\Omega, u=0$ on $\partial \Omega$, admits a unique solution $u \in C^{1}(\bar{\Omega})$ such that $\left|u^{\prime}\right|^{p-2} u^{\prime}$ is absolutely continuous and that the equation holds in the pointwise sense. (See, for example, [10, 11].)

On the other side, it is also well known that if $g \in L^{p^{\prime}}(\Omega)$ (where as usual $p^{\prime}$ is given by $\left.1 / p+1 / p^{\prime}=1\right)$ and $0 \leq c \in L^{\infty}(\Omega)$, the problem

$$
\begin{cases}-\left(\left|v^{\prime}\right|^{p-2} v^{\prime}\right)^{\prime}+c|v|^{p-2} v=g & \text { in } \Omega, \\ v=0 & \text { on } \partial \Omega,\end{cases}
$$

has a unique weak solution $v \in W_{0}^{1, p}(\Omega)$, that is, satisfying

$$
\int_{\Omega}\left|v^{\prime}\right|^{p-2} v^{\prime} \varphi^{\prime}+c|v|^{p-2} v \varphi=\int_{\Omega} g \varphi \quad \text { for all } \varphi \in W_{0}^{1, p}(\Omega)
$$

(see, for example, [7]). Furthermore, employing the comparison principles in, for instance, $[8, \mathrm{Ch} .6]$, and recalling the above paragraph, it is easy to check the following facts: $v \in C^{1}(\bar{\Omega}),\left|v^{\prime}\right|^{p-2} v^{\prime}$ is absolutely continuous and (2.1) holds a.e. $x \in \Omega$.

We say that $0 \leq v \in W_{0}^{1, p}(\Omega)$ is a (weak) subsolution of (1.1) if

$$
\int_{\Omega}\left|v^{\prime}\right|^{p-2} v^{\prime} \varphi^{\prime}+c(x) v^{p-1} \varphi \leq \int_{\Omega} m(x) u^{q} \varphi \quad \text { for all } 0 \leq \varphi \in W_{0}^{1, p}(\Omega)
$$


and $v=0$ on $\partial \Omega$; and $0 \leq w \in W_{0}^{1, p}(\Omega)$ is said to be a supersolution if (2.2) holds (with $w$ in place of $v$ ) reversing the inequality, and $w \geq 0$ on $\partial \Omega$. The well-known subsupersolution method $[5,6]$ gives a solution provided there exist a subsolution $v$ and a supersolution $w$ satisfying $v \leq w$.

REMARK 2.1. Let us write as usual $m=m^{+}-m^{-}$with $m^{+}=\max (m, 0)$ and $m^{-}=$ $\max (-m, 0)$. If $m^{+} \not \equiv 0$, one can readily verify that (1.1) admits arbitrarily large supersolutions. Indeed, let $v \geq 0$ be the solution of (2.1) with $m^{+}$in place of $g$, and let $k \geq\left(\|v\|_{\infty}+1\right)^{q /(p-1-q)}$. Then $k(v+1)$ is a supersolution since $v=k>0$ on $\partial \Omega$ and

$$
\begin{aligned}
& -\left(\left|k(v+1)^{\prime}\right|^{p-2} k(v+1)^{\prime}\right)^{\prime}+c(k(v+1))^{p-1} \\
& \quad \geq k^{p-1} m^{+} \geq\left(k\left(\|v\|_{\infty}+1\right)\right)^{q} m^{+} \geq(k(v+1))^{q} m \quad \text { in } \Omega .
\end{aligned}
$$

The next remark summarises some necessary facts about principal eigenvalues for problems with weight involving the $p$-Laplacian operator.

ReMARK 2.2. Let $0 \leq c \in L^{\infty}(\Omega)$ and let $m \in L^{p^{\prime}}(\Omega)$ with $m^{+} \not \equiv 0$. There exists a positive principal eigenvalue $\lambda_{1}(m, \Omega)$ and $\Phi \in W_{0}^{1, p}(\Omega)$ satisfying

$$
\begin{cases}-\left(\left|\Phi^{\prime}\right|^{p-2} \Phi^{\prime}\right)^{\prime}+c(x) \Phi^{p-1}=\lambda_{1}(m, \Omega) m(x) \Phi^{p-1} & \text { in } \Omega \\ \Phi>0 & \text { in } \Omega, \\ \Phi=0 & \text { on } \partial \Omega .\end{cases}
$$

Moreover, $\lambda_{1}(m, \Omega)$ is unique and simple. (See, for example, [3] and the references therein.)

\section{Main results}

In order to avoid overloading the notation, for $y \geq a, z \leq b$ and $\varepsilon \geq 0$ we set

$$
M_{a, \varepsilon}^{-}(y):=\int_{a}^{y}\left(m^{-}(x)+\varepsilon\right) d x, \quad M_{b, \varepsilon}^{-}(z):=\int_{z}^{b}\left(m^{-}(x)+\varepsilon\right) d x .
$$

If $\varepsilon=0$ we simply write $M_{a}^{-}(y)$ and $M_{b}^{-}(z)$.

THEOREM 3.1. Let $m \in L^{p^{\prime}}(\Omega)$ and suppose there exist $a \leq x_{0}<x_{1} \leq b$ with $0 \not \equiv \geq 0$ in $I:=\left(x_{0}, x_{1}\right)$. Let

$$
\gamma:=\max \left\{x_{1}-a, b-x_{0}\right\}
$$

and

$$
\mathcal{M}_{p}:=\max \left\{M_{a}^{-}\left(x_{1}\right)^{2-p}\left(\int_{a}^{x_{1}} M_{a}^{-}(x) d x\right)^{p-1}, M_{b}^{-}\left(x_{0}\right)^{2-p}\left(\int_{x_{0}}^{b} M_{b}^{-}(x) d x\right)^{p-1}\right\} .
$$

(i) Assume $p \geq 2$ and $q \in(p-2, p-1)$. If

$$
\gamma^{p-2} \mathcal{M}_{2}<\frac{p-1}{(p-1-q)^{p-1}} \frac{1}{\lambda_{1}(m, I)}
$$


and

$$
\gamma^{p}\|c\|_{L^{\infty}(\Omega)} \leq \frac{(2-p+q)(p-1)}{(p-1-q)^{p}}
$$

then there exists a solution of (1.1).

(ii) Assume $p \in(1,2]$. If

$$
\mathcal{M}_{p}<\frac{(p-1)^{p}}{(p-1-q)^{p-1}} \frac{1}{\lambda_{1}(m, I)}
$$

and

$$
\gamma^{p}\|c\|_{L^{\infty}(\Omega)} \leq\left(\frac{p-1}{p-1-q}\right)^{p} q
$$

then there exists a solution of (1.1).

Proof. Without loss of generality we assume that $a<x_{0}<x_{1}<b$. (In fact, it shall be clear from the proof how to proceed if either $x_{0}=a$ or $x_{1}=b$.) Taking into account Remark 2.1 it suffices to construct a strictly positive (in $\Omega$ ) weak subsolution $u$ for (1.1). Moreover, it is clear that it is enough to provide such subsolution for (1.1) with $\tau m$ in place of $m$, for some $\tau>0$. that

Let us prove (i). In view of (3.2) we may choose $\varepsilon>0$ small enough and fix $\tau$ such

$$
\gamma^{p-2} \frac{(p-1-q)^{p-1}}{p-1} \max \left\{\int_{a}^{x_{1}} M_{a, \varepsilon}^{-}(x) d x, \int_{x_{0}}^{b} M_{b, \varepsilon}^{-}(x) d x\right\} \leq \frac{1}{\tau} \leq \frac{1}{\lambda_{1}(m, I)} .
$$

Let $x \in\left[a, x_{1}\right]$ and define

$$
u_{1}(x):=\left(\sigma \int_{a}^{x} M_{a, \varepsilon}^{-}(y) d y\right)^{k}
$$

where

$$
k:=\frac{1}{p-1-q}, \quad \sigma:=\frac{\tau \gamma^{p-2}}{(p-1) k^{p-1}} .
$$

We have that $u_{1}(a)=0$ and that $u_{1}$ is strictly increasing. Also, from the first inequality in (3.6) it follows that $\left\|u_{1}\right\|_{\infty} \leq 1$. Let $l:=(k-1)(p-1)$. Since $q>p-2$ it holds that $l>0$. Furthermore,

$$
l-1+p=k(p-1), \quad l+p-2=k q,
$$

and by (3.3) we also obtain that $k^{p-1} l \geq \gamma^{p}\|c\|_{\infty}$. On the other hand, since $M_{a, \varepsilon}^{-}$is strictly increasing we derive that $(x-a) M_{a, \varepsilon}^{-}(x) \geq \int_{a}^{x} M_{a, \varepsilon}^{-}(y) d y$ for all $x$. Taking into account the aforementioned facts, (3.8) and that $p \geq 2$ and $x_{1}-a \leq \gamma$, some computations 
show that

$$
\begin{aligned}
&-\left(\left|u_{1}^{\prime}(x)\right|^{p-2} u_{1}^{\prime}(x)\right)^{\prime}=-\left(k \sigma^{k}\right)^{p-1}\left(l\left(\int_{a}^{x} M_{a, \varepsilon}^{-}(y) d y\right)^{l-1} M_{a, \varepsilon}^{-}(x)^{p}\right. \\
&\left.+(p-1)\left(\int_{a}^{x} M_{a, \varepsilon}^{-}(y) d y\right)^{l} M_{a, \varepsilon}^{-}(x)^{p-2}\left(m^{-}(x)+\varepsilon\right)\right) \\
& \leq-\left(k \sigma^{k}\right)^{p-1}\left(\frac{l}{\gamma^{p}}\left(\int_{a}^{x} M_{a, \varepsilon}^{-}(y) d y\right)^{k(p-1)}\right. \\
&\left.\quad+\frac{(p-1)}{\gamma^{p-2}}\left(\int_{a}^{x} M_{a, \varepsilon}^{-}(y) d y\right)^{k q} m^{-}\right) \\
& \leq-\|c\|_{\infty} \sigma^{k(p-1)}\left(\int_{a}^{x} M_{a, \varepsilon}^{-}(y) d y\right)^{k(p-1)} \\
&-\tau m^{-} \sigma^{k q}\left(\int_{a}^{x} M_{a, \varepsilon}^{-}(y) d y\right)^{k q} \\
& \leq-c u_{1}^{p-1}-\tau m^{-} u_{1}^{q} \leq-c u_{1}^{p-1}+\tau m u_{1}^{q} \quad \text { in }\left(a, x_{1}\right) .
\end{aligned}
$$

In a similar way, if for $x \in\left[x_{0}, b\right]$ we set $u_{3}$ by $u_{3}(x):=\left(\sigma \int_{x}^{b} M_{b, \varepsilon}^{-}(y) d y\right)^{k}$ with $k$ and $\sigma$ given by (3.8), then $u_{3}(b)=0, u_{3}$ is strictly decreasing, $\left\|u_{3}\right\|_{\infty} \leq 1$ and

$$
-\left(\left|u_{3}^{\prime}\right|^{p-2} u_{3}^{\prime}\right)^{\prime}+c u_{3}^{p-1} \leq \tau m u_{3}^{q} \quad \text { in }\left(x_{0}, b\right) .
$$

On the other side, let $u_{2}>0$ with $\left\|u_{2}\right\|_{L^{\infty}(I)}=1$ be the positive principal eigenfunction associated to the weight $m$ in $I$, that is, satisfying (2.3) with $I$ in place of $\Omega$. Recalling that $m \geq 0$ in $I$ and that $q<p-1$, from the second inequality in (3.6),

$$
-\left(\left|u_{2}^{\prime}\right|^{p-2} u_{2}^{\prime}\right)^{\prime}+c u_{2}^{p-1}=\lambda_{1}(m, I) m u_{2}^{p-1} \leq \tau m u_{2}^{q} \quad \text { in } I .
$$

Since

$$
u_{1}(a)=u_{3}(b)=u_{2}\left(x_{0}\right)=u_{2}\left(x_{1}\right)=0 \quad \text { and } \quad\left\|u_{1}\right\|_{\infty},\left\|u_{3}\right\|_{\infty} \leq 1=\left\|u_{2}\right\|_{\infty},
$$

arguing as in the proof of Theorem 3.1(i) in [KM] we can find $\underline{x}_{0}, \bar{x}_{1} \in I$ with $\underline{x}_{0}<\bar{x}_{1}$ and such that

$$
\begin{array}{ll}
u_{1}\left(\underline{x}_{0}\right)=u_{2}\left(\underline{x}_{0}\right), & u_{2}\left(\bar{x}_{1}\right)=u_{3}\left(\bar{x}_{1}\right), \\
u_{1}^{\prime}\left(\underline{x}_{0}\right) \leq u_{2}^{\prime}\left(\underline{x}_{0}\right), & u_{2}^{\prime}\left(\bar{x}_{1}\right) \leq u_{3}^{\prime}\left(\bar{x}_{1}\right) .
\end{array}
$$

We now define a function $u$ by $u:=u_{1}$ in $\left[a, \underline{x}_{0}\right], u:=u_{2}$ in $\left[\underline{x}_{0}, \bar{x}_{1}\right]$ and $u:=u_{3}$ in $\left[\bar{x}_{1}, b\right]$. Taking into account (3.10), a simple integration by parts yields that $u$ is a weak subsolution for (1.1) with $\tau m$ in place of $m$, and, as we said at the beginning of the proof, this proves (i).

Let us prove (ii). We first pick $\varepsilon>0$ sufficiently small and take $\tau$ such that

$$
\begin{aligned}
& \frac{(p-1-q)^{p-1}}{(p-1)^{p}}\left(\int_{a}^{x_{1}} M_{a, \varepsilon}^{-}(x) d x\right)^{p-1} \leq \frac{1}{\tau} \leq \frac{1}{M_{a, \varepsilon}^{-}\left(x_{1}\right)^{2-p} \lambda_{1}(m)}, \\
& \frac{(p-1-q)^{p-1}}{(p-1)^{p}}\left(\int_{x_{0}}^{b} M_{b, \varepsilon}^{-}(x) d x\right)^{p-1} \leq \frac{1}{\tau} \leq \frac{1}{M_{b, \varepsilon}^{-}\left(x_{0}\right)^{2-p} \lambda_{1}(m)} .
\end{aligned}
$$


(This is possible due to (3.4).) Let $M_{\varepsilon}:=\max \left\{M_{a, \varepsilon}^{-}\left(x_{1}\right), M_{b, \varepsilon}^{-}\left(x_{0}\right)\right\}$. We shall build a strictly positive subsolution for (1.1) with $\tau M_{\varepsilon}^{p-2} m$ in place of $m$. For $x \in\left[a, x_{1}\right]$ we set $u_{1}$ as in (3.7) with

$$
k:=\frac{p-1}{p-1-q}, \quad \sigma:=\frac{1}{k}\left(\frac{\tau}{p-1}\right)^{1 /(p-1)}
$$

in place of (3.8). Again $u_{1}(a)=0, u_{1}$ is strictly increasing and using the first inequality in (3.11) one can check that $\left\|u_{1}\right\|_{\infty} \leq 1$. Taking $l$ as in (i) we now obtain $l=k q$ and also as before we have $l-1+p=k(p-1)$ and $k^{p-1} l \geq \gamma^{p}\|c\|_{\infty}$. Furthermore, recalling that $p \leq 2$ and arguing as in (3.9), we deduce that

$$
\begin{aligned}
-\left(\left|u_{1}^{\prime}(x)\right|^{p-2} u_{1}^{\prime}(x)\right)^{\prime} \leq & -\left(k \sigma^{k}\right)^{p-1}\left(\frac{l}{\gamma^{p}}\left(\int_{a}^{x} M_{a, \varepsilon}^{-}(y) d y\right)^{l-1+p}\right. \\
& \left.+(p-1)\left(\int_{a}^{x} M_{a, \varepsilon}^{-}(y) d y\right)^{l} M_{a, \varepsilon}^{-}\left(x_{1}\right)^{p-2} m^{-}\right) \\
\leq & -\left(k \sigma^{k}\right)^{p-1}\left(\frac{l}{\gamma^{p}}\left(\int_{a}^{x} M_{a, \varepsilon}^{-}(y) d y\right)^{k(p-1)}\right. \\
& \left.\quad+(p-1)\left(\int_{a}^{x} M_{a, \varepsilon}^{-}(y) d y\right)^{k q} M_{\varepsilon}^{p-2} m^{-}\right) \\
\leq & -\|c\|_{\infty} \sigma^{k(p-1)}\left(\int_{a}^{x} M_{a, \varepsilon}^{-}(y) d y\right)^{k(p-1)} \\
& \quad-\tau M_{\varepsilon}^{p-2} m^{-} \sigma^{k q}\left(\int_{a}^{x} M_{a, \varepsilon}^{-}(y) d y\right)^{k q} \\
\leq- & c u_{1}^{p-1}-\tau M_{\varepsilon}^{p-2} m^{-} u_{1}^{q} \\
\leq & -c u_{1}^{p-1}+\tau M_{\varepsilon}^{p-2} m u_{1}^{q} \text { in }\left(a, x_{1}\right) .
\end{aligned}
$$

Since $u_{3}$ can be defined analogously and, taking into account the definition of $M_{\varepsilon}$ and the second inequality in (3.11) and (3.12), $u_{2}$ can be chosen as above (that is, as the normalised positive principal eigenfunction with respect to the weight $m$ in $I$ ), arguing as in (i), the theorem follows.

\section{REMARK 3.2.}

(i) Let us note that when $m \in C(\Omega)$ the condition $0 \not \equiv m \geq 0$ in $I$ is necessary to have a (nontrivial) nonnegative solution for (1.1).

(ii) Let us also observe that if $p=2$ then (3.2) and (3.3) coincide with (3.4) and (3.5) and that the resulting conditions extend the ones in $[\mathrm{KM}]$ (see Theorem 3.5(ii) there).

For $p>1$ and $q \in(0, p-1)$ we set

$$
C_{p, q}:=\left(\frac{p}{p-1-q}\right)^{p-1} \frac{(p-1)(q+1)}{p-1-q} .
$$


We point out that for any $p>1, \lim _{q \rightarrow p-1^{-}} C_{p, q}=\infty$. We shall now assume that $m^{-} \in L^{\infty}(\Omega)$. In the following theorem we suppose that $c \equiv 0$. The case $c \equiv 0$ is considered in Corollary 3.5 below.

THeOREm 3.3. Assume $c \not \equiv 0$. Let $m \in L^{p^{\prime}}(\Omega)$ with $m^{-} \in L^{\infty}(\Omega)$ and suppose there exist $a \leq x_{0}<x_{1} \leq b$ such that $0 \neq m \geq 0$ in $I:=\left(x_{0}, x_{1}\right)$. Let $\gamma$ and $C_{p, q}$ be given by (3.1) and (3.13), respectively.

(i) Assume $p \geq 2$. If

$$
\frac{\left\|m^{-}\right\|_{L^{\infty}(\Omega)}}{\|c\|_{L^{\infty}(\Omega)}} \sinh ^{p}\left(\left(\frac{\|c\|_{L^{\infty}(\Omega)}}{C_{p, q}}\right)^{1 / p} \gamma\right) \leq \frac{1}{\lambda_{1}(m, I)}
$$

then there exists a solution of (1.1).

(ii) Assume $p \in(1,2)$. If

$$
\frac{\left\|m^{-}\right\|_{L^{\infty}(\Omega)}}{\|c\|_{L^{\infty}(\Omega)}}\left(e^{\left(\|c\|_{L^{\infty}(\Omega)} / C_{p, q}\right)^{1 / p} \gamma}-1\right)^{p} \leq \frac{1}{\lambda_{1}(m, I)}
$$

then there exists a solution of (1.1).

Proof. The proof follows the lines of the proof of Theorem 3.1 and hence we omit the details. We only indicate briefly how to construct $u_{1}$ in both (i) and (ii). Suppose first (3.14) holds. Let $\tau$ be such that

$$
\frac{\left\|m^{-}\right\|_{L^{\infty}(\Omega)}}{\|c\|_{L^{\infty}(\Omega)}} \sinh ^{p}\left(\left(\frac{\|c\|_{L^{\infty}(\Omega)}}{C_{p, q}}\right)^{1 / p} \gamma\right) \leq \frac{1}{\tau} \leq \frac{1}{\lambda_{1}(m, I)}
$$

and for $x \in\left[a, x_{1}\right]$ define

$$
f(x):=\left(\frac{\tau\left\|m^{-}\right\|_{\infty}}{\|c\|_{\infty}}\right)^{1 / p} \sinh \left(\left(\frac{\|c\|_{\infty}}{C_{p, q}}\right)^{1 / p}(x-a)\right) .
$$

It is easy to check that $\left(C_{p, q}^{1 / p} f^{\prime}\right)^{2}-\left(\|c\|_{\infty}^{1 / p} f\right)^{2}=\left(\tau\left\|m^{-}\right\|_{\infty}\right)^{2 / p}$ in $\left(a, x_{1}\right)$. Moreover, $f(a)=0, f$ is increasing (in particular, employing (3.16) and the fact that $x_{1}-a \leq \gamma$, we see that $\left.\|f\|_{\infty} \leq 1\right)$, and $f^{\prime \prime} \geq 0$ in $\left(a, x_{1}\right)$. Let us now choose

$$
k:=\frac{p}{p-1-q}, \quad l:=(k-1)(p-1) .
$$

Then $l-1=k q, l-1+p=k(p-1)$ and $k^{p-1} l=C_{p, q}$. Define $u_{1}:=f^{k}$. Taking into account the above mentioned facts and that $p \geq 2$, we find that, in $\left(a, x_{1}\right)$,

$$
\begin{aligned}
-\left(\left|u_{1}^{\prime}\right|^{p-2} u_{1}^{\prime}\right)^{\prime}+c u^{p-1} \leq & -k^{p-1}\left(l f^{l-1}\left(f^{\prime}\right)^{p}+(p-1) f^{l}\left(f^{\prime}\right)^{p-2} f^{\prime \prime}\right) \\
& +\|c\|_{\infty} f^{k(p-1)} \\
\leq & -k^{p-1} l f^{l-1}\left(f^{\prime}\right)^{p}+\|c\|_{\infty} f^{k(p-1)} \\
= & -f^{l-1}\left(C_{p, q}\left(f^{\prime}\right)^{p}-\|c\|_{\infty} f^{p}\right) \\
\leq & -f^{l-1}\left(\left(C_{p, q}^{1 / p} f^{\prime}\right)^{2}-\left(\|c\|_{\infty}^{1 / p} f\right)^{2}\right)^{p / 2} \\
& =-f^{l-1} \tau\left\|m^{-}\right\|_{\infty} \leq \tau m u_{1}^{q} .
\end{aligned}
$$


Suppose now (3.15) holds. In this case we take $\tau$ and $f$ such that

$$
\frac{\left\|m^{-}\right\|_{L^{\infty}(\Omega)}}{\|c\|_{L^{\infty}(\Omega)}}\left(e^{\left(\|c\|_{L^{\infty}(\Omega)} / C_{p, q}\right)^{1 / p} \gamma}-1\right)^{p} \leq \frac{1}{\tau} \leq \frac{1}{\lambda_{1}(m, I)},
$$

and

$$
f(x):=\sigma\left(e^{\lambda(x-a)}-1\right)
$$

where

$$
\sigma:=\left(\frac{\tau\left\|m^{-}\right\|_{\infty}}{\|c\|_{\infty}}\right)^{1 / p}, \quad \lambda:=\left(\frac{\|c\|_{\infty}}{C_{p, q}}\right)^{1 / p} .
$$

Let $k$ and $l$ be given by (3.17), and let $u_{1}:=f^{k}$. Reasoning as in (3.18) yields

$$
\begin{aligned}
-\left(\left|u_{1}^{\prime}\right|^{p-2} u_{1}^{\prime}\right)^{\prime}+c u^{p-1} & \leq-f^{l-1}\left(C_{p, q}\left(f^{\prime}\right)^{p}-\|c\|_{\infty} f^{p}\right) \\
& =-f^{l-1}\left(C_{p, q}(\sigma \lambda)^{p} e^{p \lambda(x-a)}-\|c\|_{\infty} \sigma^{p}\left(e^{\lambda(x-a)}-1\right)^{p}\right) \\
& \leq-f^{l-1}\|c\|_{\infty} \sigma^{p} \\
& =-f^{l-1} \tau\left\|m^{-}\right\|_{\infty} \leq \tau m u_{1}^{q}
\end{aligned}
$$

in $\left(a, x_{1}\right)$.

REMARK 3.4. A quick look at the proof of the above theorem shows that (ii) holds for any $p>1$. We observe however that one can verify that the inequality (3.14) is better than (3.15).

COROLlary 3.5. Let $m$ be as in the above theorem and suppose $c \equiv 0$. If

$$
\frac{\left\|m^{-}\right\|_{L^{\infty}(\Omega)} \gamma^{p}}{C_{p, q}} \leq \frac{1}{\lambda_{1}(m, I)}
$$

then there exists a solution of (1.1).

Proof. It is enough to note that the left side of either (3.14) or (3.15) tends to the left side of (3.19) when $\|c\|_{\infty}$ goes to zero. Alternatively, one can also proceed as in the proof of the above theorem taking (for any $p>1) f(x):=(x-a) / \gamma$.

REMARK 3.6. Let us suppose that $c$ changes sign in $\Omega$. An inspection of the proofs of the theorems shows that one can still argue in the same way as before, replacing $c$ by $c^{+}$to construct the functions $u_{1}$ and $u_{3}$. Furthermore, if the positive principal eigenvalue $\lambda_{1}(m, I)$ exists (for necessary and sufficient conditions on this question, see [3, Section 2]) and if the problem (2.1) with $m^{+}$in place of $g$ admits a nonnegative solution, then all the analogous results to the case $c \geq 0$ can be proved allowing $c$ to change sign in $\Omega$.

\section{Acknowledgement}

The first author would kindly like to dedicate this work to his teacher and friend Tomás Godoy. 


\section{References}

[1] L. Boccardo and L. Orsina, 'Sublinear equations in $L^{s}$, Houston J. Math. 20 (1994), 99-114.

[2] J. Chaparova and N. Kutev, 'Positive solutions of the generalized Emden-Fowler equation in Hölder spaces', J. Math. Anal. Appl. 352 (2009), 65-76.

[3] M. Cuesta and H. Ramos Quoirin, 'A weighted eigenvalue problem for the $p$-Laplacian plus a potential', NoDEA Nonlinear Differential Equations Appl. 16 (2009), 469-491.

[4] J. Díaz, J. Hernández and F. Mancebo, 'Branches of positive and free boundary solutions for some singular quasilinear elliptic problems', J. Math. Anal. Appl. 352 (2009), 449-474.

[5] P. Drábek and J. Hernández, 'Existence and uniqueness of positive solutions for some quasilinear elliptic problems', Nonlinear Anal. 44 (2001), 189-204.

[6] Y. Du, Order Structure and Topological Methods in Nonlinear Partial Differential Equations. Vol. 1. Maximum Principles and Applications, Series in Partial Differential Equations and Applications, 2 (World Scientific Publishing Co Ltd, Hackensack, NJ, 2006).

[7] J. García-Melián and J. Sabina de Lis, 'Maximum and comparison principles for operators involving the $p$-Laplacian', J. Math. Anal. Appl. 218 (1998), 49-65.

[8] L. Gasiński and N. Papageorgiou, Nonlinear Analysis, Series in Mathematical Analysis and Applications, 9 (Chapman \& Hall, Boca Raton, FL, 2006).

[9] T. Godoy and U. Kaufmann, 'On strictly positive solutions for some semilinear elliptic problems', NoDEA Nonlinear Differential Equations Appl. 20 (2013), 779-795.

[10] R. Manásevich and J. Mawhin, 'Periodic solutions for nonlinear systems with $p$-Laplacian-like operators', J. Differential Equations 145 (1998), 367-393.

[11] R. Manásevich and J. Mawhin, 'Boundary value problems for nonlinear perturbations of vector p-Laplacian-like operators', J. Korean Math. Soc. 37 (2000), 665-685.

U. KAUFMANN, FaMAF, Universidad Nacional de Córdoba, (5000) Córdoba, Argentina

e-mail: kaufmann@mate.uncor.edu

I. MEDRI, FaMAF, Universidad Nacional de Córdoba, (5000) Córdoba, Argentina

e-mail: medri@mate.uncor.edu 